\title{
Circular RNA expression profiles in the plasma of patients with infantile hemangioma determined using microarray analysis
}

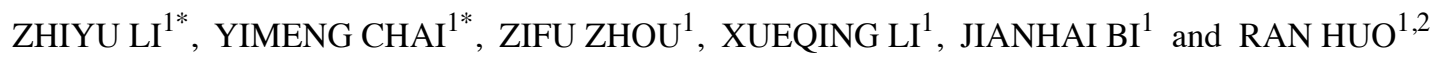 \\ ${ }^{1}$ Department of Plastic and Aesthetic Surgery, Shandong Provincial Hospital, Cheeloo College of \\ Medicine, Shandong University; ${ }^{2}$ Department of Plastic and Aesthetic Surgery, Shandong Provincial \\ Hospital Affiliated to Shandong First Medical University, Jinan, Shandong 250021, P.R. China
}

Received August 21, 2020; Accepted March 3, 2021

DOI: $10.3892 /$ etm.2021.10066

\begin{abstract}
Circular RNAs (circRNAs) are noncoding RNAs that have important roles in tumor progression. Previous studies have examined the circRNAs involved in infantile hemangioma (IH) tumors. The present study compared the circRNA levels in plasma samples from patients with IH and control individuals. The circRNA expression profiles were determined using microarray in three pairs of plasma samples from patients with proliferative $\mathrm{IH}$ and healthy control subjects. Expression of the identified differentially expressed circRNAs was verified using reverse transcription-quantitative PCR (RT-qPCR) and a bioinformatics analysis was performed to predict the microRNAs targeted by the validated circRNAs. From the circRNA expression profiles in the plasma of patients with IHs, 128 differentially expressed circRNAs were identified, of which 72 were upregulated and 56 were downregulated. The downregulated expression of three circRNAs [Homo sapiens (hsa)_circRNA_101566, hsa_circRNA_103546 and hsa_circRNA_103573] was verified using RT-qPCR. Gene Ontology term and Kyoto Encyclopedia of Genes and Genomes pathway analyses indicated that all identified networks participated in angiogenesis and tumor formation and progression. It was determined that hsa_circRNA_101566, which is able to regulate the mTOR signaling pathway, may be an important regulatory molecule in IH development and that targeting of hsa_miR_520c is able to indirectly regulate the vascular endothelial growth factor
\end{abstract}

Correspondence to: Dr Jianhai Bi or Dr Ran Huo, Department of Plastic and Aesthetic Surgery, Shandong Provincial Hospital, Cheeloo College of Medicine, Shandong University, 324 Jingwu Road, Huaiyin, Jinan, Shandong 250021, P.R. China

E-mail: bijianhai@126.com

E-mail: huoran@medmail.com.cn

${ }^{*}$ Contributed equally

Key words: circular RNAs, infantile hemangioma, microarray analysis, microRNA, coding potential signaling pathway. Further studies are required to clarify these effects and the underlying mechanisms.

\section{Introduction}

Infantile hemangioma (IH) is the most common type of soft tissue tumor in infants, with an incidence of $4-10 \%(1,2)$. The growth of IH typically increases rapidly during the first three months, particularly between the first five and eight weeks of life, and $\sim 80 \%$ of its maximum growth is completed by the age of three months $(3,4)$. Although most IHs resolve naturally without treatment, $10-15 \%$ of cases have complications, such as obstruction, ulceration or disfigurement, and require intervention. IH results from angiogenesis and angiogenesis-related disorders. However, the factors that trigger IH development have remained to be elucidated. Therefore, studies investigating the molecular mechanisms of the tumorigenesis and progression of IH are required.

Circular RNA (circRNA) is a type of noncoding RNA (ncRNA) composed of transcripts from exons. It was first discovered by Sanger et al (5) in a virus by using electron microscopy in 1976. Over the last few years, circRNAs have been indicated to have multiple functions, including acting as microRNA (miRNA/miR) sponges, influencing gene expression by regulating splicing or transcription and interacting with RNA-binding proteins (6-8). An increasing number of studies have demonstrated the functions of circRNAs in various diseases. Furthermore, circRNAs may serve as prognostic and diagnostic biomarkers for various diseases (9). A previous study by our group, in which the profiles of circRNAs in IH and adjacent normal tissues were compared, revealed that circRNAs participate in angiogenesis and vascular development-related biological processes (10). However, to the best of our knowledge, plasma circRNAs have so far remained to be systematically evaluated in IH. As circRNAs are excreted from cells in exosomes, their recovery from the blood is a viable option.

The present study aimed to determine the circRNA profiles of three pairs of plasma samples from patients with proliferative $\mathrm{IH}$ and healthy control individuals using high-throughput microarray and explore the roles of these circRNAs in $\mathrm{IH}$ using bioinformatic methods. The results enhance the current understanding of the roles of circRNAs in the pathogenesis of 
$\mathrm{IH}$ and may aid in the discovery of blood-based biomarkers for diagnosing IH.

\section{Materials and methods}

Patient samples. In the present study, the inclusion criteria were as follows: Proliferative IH diagnosed by two independent doctors according to clinical and pathological features (red soft lump that appears within 3 months of birth and rapidly increases in size, with an elevated skin temperature) (3) and Doppler ultrasound (frequently subcutaneous, with variable echogenicity; intralesional vessels not visible on gray-scale imaging; high vascular density on color Doppler; increased echogenicity and decreased vascularity with involution) (11). Furthermore, only patients aged 3-6 months were considered to ensure that the IHs were still in the proliferative phase. The exclusion criteria were as follows: i) Administration of any treatment for IH prior to participating in the study and ii) the presence of another disease. Blood samples from a total of 17 patients with IH were collected at the Department of Plastic Surgery of Shandong Provincial Hospital (Jinan, China) between May 2018 and April 2019. In addition, blood samples were collected from 17 normal age-matched healthy volunteers. The volunteers came from the children in the physical examination center of Shandong Provincial Hospital (Jinan, China) and blood was collected with the consent of their parents/guardians. Plasma was obtained from the blood samples via centrifugation and stored at $-80^{\circ} \mathrm{C}$. A total of three randomly selected sample pairs were used for microarray analysis. The other 14 sample pairs were used for further validation of the microarray results. The demographic characteristics of the cohort and the clinicopathological features of the patients with IH are provided in Table I.

RNA isolation and quality control. RNA from each sample was isolated using TRIzol reagent (Invitrogen; Thermo Fisher Scientific, Inc.) according to the manufacturer's protocol. The purity and concentration of the total RNA samples were determined using a NanoDrop ND-1000 spectrometer (NanoDrop Technologies; Thermo Fisher Scientific, Inc.). RNA integrity was assessed through electrophoresis on a denaturing agarose gel.

Microarray. Sample labeling and array hybridization were performed according to the manufacturer's protocol (Arraystar human circular RNA v2; design ID 074301). In brief, total RNAs were digested with RNase R (Epicentre; Illumina, Inc.) to remove linear RNAs and enrich circRNAs. The enriched circRNAs were amplified and transcribed into fluorescent complementary (c)RNAs using a random priming method according to the Arraystar's protocol (Super RNA Labeling Kit; Arraystar, Inc.). The labeled cRNAs were purified using an RNeasy Mini Kit (Qiagen GmbH). The concentration and specific activity of the labeled cRNAs (pmol Cy3/ $\mu \mathrm{g}$ cRNA) were measured using a NanoDrop ND-1000. Thereafter, $1 \mu \mathrm{g}$ of each labeled cRNA was fragmented by adding $5 \mu \mathrm{l}$ of 10X blocking agent and $1 \mu \mathrm{l}$ of $25 \mathrm{X}$ fragmentation buffer, followed by heating at $60^{\circ} \mathrm{C}$ for $30 \mathrm{~min}$. Subsequently, $25 \mu 1$ of $2 \mathrm{X}$ hybridization buffer was added to dilute the labeled cRNA.
Next, $50 \mu 1$ of hybridization solution was dispensed into the gasket slide and assembled on the circRNA expression microarray slide. The slides were incubated for $17 \mathrm{~h}$ at $65^{\circ} \mathrm{C}$ in an Agilent Hybridization Oven (Agilent Technologies, Inc.). The hybridized arrays were washed, fixed and scanned using an Agilent Scanner (G2505C; Agilent Technologies, Inc.).

Data analysis. Raw data were extracted using the Agilent Feature Extraction software (version 11.0.1.1; Agilent Technologies, Inc.). A series of data-processing steps, including quantile normalization and low-intensity filtering, were performed using the limma package of the $\mathrm{R}$ software (https://rstudio.com/). The fold-change (FC; i.e., ratio of group averages) in the plasma circRNA levels between patients with IH and normal subjects was determined for each circRNA. The statistical significance of the differences was estimated using the Student's t-test. circRNAs with $\mathrm{FCs} \geq 1.5$ and $\mathrm{P} \leq 0.05$ were considered to be significantly differentially expressed.

Validation of microarray data using reverse transcription-quantitative PCR (RT-qPCR). The total RNA from the three paired plasma samples of IH cases and healthy children used for microarray analysis was isolated using the TRIzol reagent and reverse-transcribed (2X PCR master mix; Arraystar, Inc.; $95^{\circ} \mathrm{C}$ for $10 \mathrm{~min}$, followed by 40 cycles of $95^{\circ} \mathrm{C}$ for $10 \mathrm{sec}$ and $60^{\circ} \mathrm{C}$ for $60 \mathrm{sec}$ ) to synthesize cDNA using a GeneAmp PCR System 9700 (Applied Biosystems; Thermo Fisher Scientific, Inc.) according to the kit's protocols. GAPDH was selected as the internal reference. All cDNAs were assembled in the ViiA 7 real-time PCR System (Applied Biosystems; Thermo Fisher Scientific, Inc.) (2X Master Mix, $5 \mu \mathrm{l} ; 10 \mu \mathrm{M}$ PCR specific primer $\mathrm{F}, 0.5 \mu \mathrm{l} ; 10 \mu \mathrm{M}$ PCR specific primer R, $0.5 \mu \mathrm{l}$; water was added to create the total volume of $8 \mu \mathrm{l})$. The reaction was performed as follows: $95^{\circ} \mathrm{C}$ for $10 \mathrm{~min}$, followed by 40 cycles of $95^{\circ} \mathrm{C}$ for $10 \mathrm{sec}$ and $60^{\circ} \mathrm{C}$ for $60 \mathrm{sec}$. All targets and references were evaluated in triplicate wells. The relative expression levels of each target circRNA were calculated using the $2^{-\Delta \Delta C q}$ method (12). Primer sequences are presented in Table II. The identified circRNAs, which were consistent with the regulatory direction of the microarray results and significantly differentially expressed, were further verified in the remaining 14 sample pairs.

Construction of circRNA-miRNA-mRNA networks. The circRNA-targeted miRNAs were predicted using Arraystar's homemade miRNA target prediction software (Arraystar, Inc.) based on the data obtained from TargetScan, miRanda and statistical analysis (methods listed in Data S1). The network was constructed using Cytoscape software (version 2.8.3) (https://cytoscape.org/). Gene ontology (GO) term analysis was performed using the Database for Annotation, Visualization and Integrated Discovery (DAVID) tool (http://david.abcc. ncifcrf.gov/) to explore the potential functions of the target genes in the categories biological process, cellular component and molecular function. The Kyoto Encyclopedia of Genes and Genomes (KEGG) is a database resource for understanding the high-level functions and effects of a biological system. KEGG pathway enrichment analysis was performed using the KEGG Orthology-Based Annotation System (KOBAS; http://kobas. cbi.pku.edu.cn/). 
Table I. Baseline characteristics of patients with IH and control subjects.

\begin{tabular}{lrrr}
\hline Characteristic & IH $(\mathrm{n}=17)$ & Control (n=17) \\
\hline $\begin{array}{l}\text { Sex } \\
\text { Male }\end{array}$ & 6 & & \\
Female & 11 & $(64.3)$ & $6(35.3)$ \\
Age (months) & & & $11(64.7)$ \\
$<3$ & 12 & $(70.6)$ & $11(64.7)$ \\
$\geq 3$ & 5 & $(29.4)$ & $6(35.3)$ \\
History of treatment & & & \\
Yes & 0 & $(0)$ & \\
No & $17(100.0)$ & \\
Location of IH & & & \\
Head, face, neck & 6 & $(35.3)$ & \\
Extremity & 3 & $(17.6)$ & \\
Trunk & 6 & $(35.3)$ & \\
Multiple sites & 2 & $(11.8)$ & \\
\end{tabular}

Values are expressed as $\mathrm{n}(\%)$. IH, infantile hemangioma.

Statistical analysis. All data were expressed as the mean \pm standard error of the mean or $\mathrm{n}(\%)$ and analyzed using the Student's t-test with SPSS 22.0 software (IBM Corp.). GraphPad Prism 8 (GraphPad Software, Inc.) and Microsoft Excel 2019 (Microsoft Corp.) were used for other statistical analyses. $\mathrm{P}<0.05$ was considered to indicate statistical significance.

\section{Results}

Demographic characteristics of patients and volunteers. In the present study, 17 patients with IH (6 males and 11 females) and 17 age-matched healthy volunteers (6 males and 11 females) were collected. Among the patients with IH, 12 cases were $<3$ months old and 5 cases were $\geq 3$ months old. Among the volunteers, 11 cases were $<3$ months old and 6 were $\geq 3$ months old. The sex and age distribution of patients and volunteers were not statistically different.

Differentially expressed circRNAs detected using microarray analysis. A microarray analysis of 13,617 circRNAs in the plasma of patients with IH and healthy controls was performed. Relevant data were uploaded to the Gene Expression Omnibus (GEO) database (accession no. GSE162905). A box plot was used to indicate the intensity values of the six samples after normalization (Fig. 1A). The scatter plot (Fig. 1B) and volcano plot (Fig. 1C) revealed differences in circRNA expression between the test and control groups. Finally, data analysis and screening based on $\mathrm{FC}>1.5$ and $\mathrm{P}<0.05$ revealed 72 upregulated and 56 downregulated circRNAs. Among the upregulated circRNAs, 48 were exonic, 15 were intronic, 5 were sense overlapping, 3 were intergenic and 1 was antisense. The downregulated circRNAs included 51 exonic, 2 intronic and 3 sense overlapping circRNAs. In addition, the distribution of these circRNAs on chromosomes was summarized, indicating that they were distributed on each chromosome. The distinct differential expression of these 128 circRNAs was visualized by hierarchical clustering in the heat map (Fig. 1D). These results indicated that the present differential expression profile was reliable.

$R T-q P C R$ verification of the selected differentially expressed circRNAs. Based on known data of microarray analysis, 10 circRNAs we selected for RT-qPCR validation, including 6 upregulated and 4 downregulated circRNAs that were exonic $(\mathrm{FC}>1.5, \mathrm{P}<0.05$, raw intensity of the samples were $>100$ and the number of bases was 200-3,000; Table III). The original signal intensity was high and the number of bases was 200-3,000. The results suggested that the three downregulated circRNAs [Homo sapiens (hsa)_circRNA_101566, hsa_circRNA_103546 and hsa_circRNA_103573] were significantly differentially expressed (Fig. 2A), consistent with the microarray results. However, no upregulated circRNAs were detected. Analysis of another seven samples revealed similar expression levels or no significant differences between patients with IH and healthy children. The three circRNAs were further verified using the remaining 14 sample pairs and significantly different expression levels were detected (Fig. 2B).

MiRNA prediction and bioinformatics analysis of circRNAs. Based on the results of RT-qPCR, the miRNA response elements of the circRNAs were predicted using the Arraystar miRNA target prediction software. To further investigate the functions of these target genes, GO and KEGG analyses of the differentially expressed circRNAs were performed using DAVID and KOBAS. Based on the RT-qPCR results, three downregulated circRNAs (hsa_circRNA_101566, hsa_circRNA_103546 and hsa_circRNA_103573) were selected for further analysis. The top 10 significant pathways in the 3 categories of the GO analysis (biological process, molecular function and cellular component), including positive regulation of cell death, regulation of TOR signaling and ncRNA metabolic process, are presented in Fig. 3A. The KEGG analysis revealed the top 10 pathways associated with the downregulated circRNAs (Fig. 3B), including oxidative phosphorylation, citrate cycle, CoA biosynthesis pathway and NOD-like receptor signaling pathway. These pathways have a vital role in the development of angiogenesis and vascular diseases.

Construction of the circRNA-miRNA-mRNA network. To further evaluate the relationship between the confirmed differentially expressed circRNAs and the corresponding miRNAs and to investigate the mechanisms underlying IH development, Cytoscape was used to illustrate the hsa_circRNA_101566/hsa_circRNA_ 103546/hsa_circRNA_103573-miRNA-mRNA network (Fig.4).The number of genes targeted by hsa_circRNA_101566 was the highest among all circRNAs evaluated (Fig. 4). As the amount of raw data was large, miRNAs related to angiogenesis and partially related genes were selected (referring to the increase of other tumors' angiogenesis while promoting the growth of other tumors, so its influence on blood vessels may be indirectly regulated), including hsa_miR_765, 
Table II. Sequences of primers used for PCR.

\begin{tabular}{|c|c|c|c|}
\hline Gene name & Bidirectional primer sequence & $\begin{array}{c}\text { Annealing } \\
\text { temperature }\left({ }^{\circ} \mathrm{C}\right)\end{array}$ & $\begin{array}{c}\text { Product } \\
\text { length (bp) }\end{array}$ \\
\hline \multirow[t]{2}{*}{ GAPDH (human) } & F: 5'GGGAAACTGTGGCGTGAT3' & & \\
\hline & R: 5'GAGTGGGTGTCGCTGTTGA3' & 60 & 299 \\
\hline \multirow[t]{2}{*}{ hsa_circRNA_001490 } & F: 5'AACTCATACTATGGGTGGTGACTT3' & & \\
\hline & R: 5'CACATATCCTATGTTCATCAATCTG3' & 60 & 93 \\
\hline \multirow[t]{2}{*}{ hsa_circRNA_103372 } & F: 5'AGAAGGCAGCCAACCAGAT3' & & \\
\hline & R: 5'TTCAGATTTCTTTAGCCACTCAA3' & 60 & 120 \\
\hline \multirow[t]{2}{*}{ hsa_circRNA_103361 } & F: 5'TTTAACTAGCACTGCTTGTCGGA3' & & \\
\hline & R: 5'ATCACCTTTGGCAGGATCTTCA3' & 60 & 84 \\
\hline \multirow[t]{2}{*}{ hsa_circRNA_104310 } & F: 5'GGACACGGTCTTTCTTATTCAGG3 ' & & \\
\hline & R: 5'ACAGTGATGGTCGAAACGGTG3' & 60 & 63 \\
\hline \multirow[t]{2}{*}{ hsa_circRNA_023016 } & F: 5'TGTTGGATTTGGGAATGACCT3' & & \\
\hline & R: 5'CCACACACATCCCACTACCCT3' & 60 & 147 \\
\hline \multirow[t]{2}{*}{ hsa_circRNA_102101 } & F: 5'TTGTTGGGACATGTATATTGTACAC3' & & \\
\hline & R: 5'CTATATGCTTTATATGCCTTTCCTG3' & 60 & 93 \\
\hline \multirow[t]{2}{*}{ hsa_circRNA_103546 } & F: 5'GAGTGCAGCTCCCCCTATAAG3' & & \\
\hline & R: 5'CCCTGTACTTTGAAAGCCTCTT3' & 60 & 151 \\
\hline \multirow[t]{2}{*}{ hsa_circRNA_062683 } & F: 5'GCTGTCCTCCACCATGAAGA 3' & & \\
\hline & R: 5'CCAGGCTCACATCTCCCTT3' & 60 & 140 \\
\hline \multirow[t]{2}{*}{ hsa_circRNA_103573 } & F: 5'CGGTGTTGTTCTTTGCCATTT3' & & \\
\hline & R: 5'TGCAGGGGAATGATGAATACAG3 ' & 60 & 96 \\
\hline \multirow[t]{2}{*}{ hsa_circRNA_101566 } & F: 5'TTATACCCAGTAAAGGAGAAAGC3' & & \\
\hline & R: 5'CCTCAGAGTTTGAGAAATAACATAC3' & 60 & 91 \\
\hline
\end{tabular}

F, forward; R, reverse; circRNA, circular RNA; hsa, Homo sapiens.

Table III. Selected differentially expressed circRNAs.

\begin{tabular}{|c|c|c|c|c|c|}
\hline circRNA & P-value & Absolute FC & Direction of regulation & Chromosome & circRNA type \\
\hline hsa_circRNA_001490 & 0.023331583 & 4.1779908 & Up & 5 & Exonic \\
\hline hsa_circRNA_103372 & 0.044010096 & 3.4234124 & Up & 3 & Exonic \\
\hline hsa_circRNA_103361 & 0.036601471 & 2.7282583 & Up & 3 & Exonic \\
\hline hsa_circRNA_104310 & 0.020127023 & 2.6489823 & Up & 7 & Exonic \\
\hline hsa_circRNA_062683 & 0.045776034 & 2.1446458 & Up & 22 & Exonic \\
\hline hsa_circRNA_023016 & 0.017327291 & 2.0521739 & Up & 11 & Exonic \\
\hline hsa_circRNA_102101 & 0.043224524 & 3.961216 & Down & 17 & Exonic \\
\hline hsa_circRNA_103546 & 0.039832007 & 2.2214431 & Down & 3 & Exonic \\
\hline hsa_circRNA_103573 & 0.032726148 & 1.8586376 & Down & 3 & Exonic \\
\hline hsa_circRNA_101566 & 0.022844096 & 1.703774 & Down & 15 & Exonic \\
\hline
\end{tabular}

FC, fold change; circRNA, circular RNA; hsa, Homo sapiens.

hsa_miR_486, hsa_miR_18a, hsa_miR_10a, hsa_miR_223 and hsa_miR_522 (13-20). The remaining targets are listed in Table SI. It was indicated that hsa_circRNA_101566, hsa_circRNA_103546 and hsa_circRNA_103573 contain $18, \overline{4}$ and 3 potential miRNA response elements, respectively (Fig. 4).

\section{Discussion}

To the best of our knowledge, the present study was the first study to detect and profile circRNAs in the plasma of patients with IH. The results of the present study revealed that the downregulated circRNAs, namely hsa_circRNA_101566, 
A

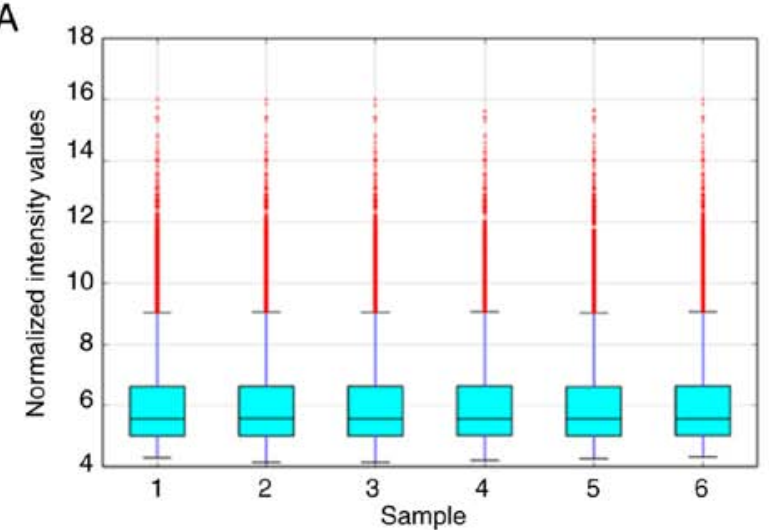

C

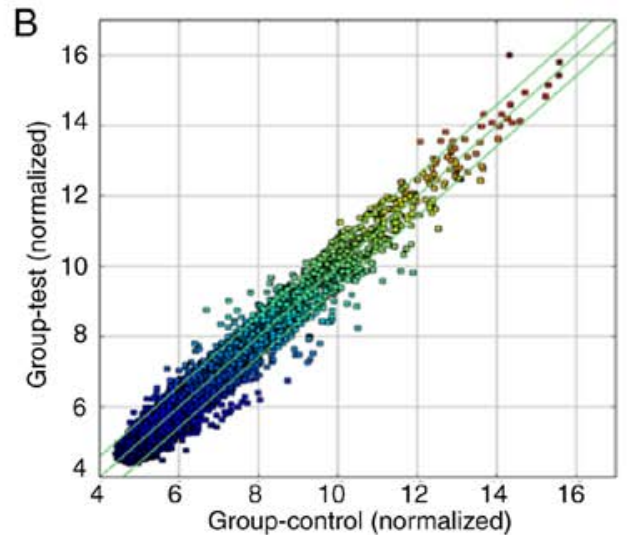

B

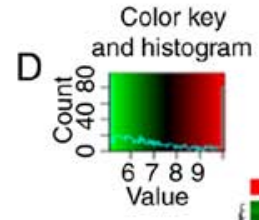

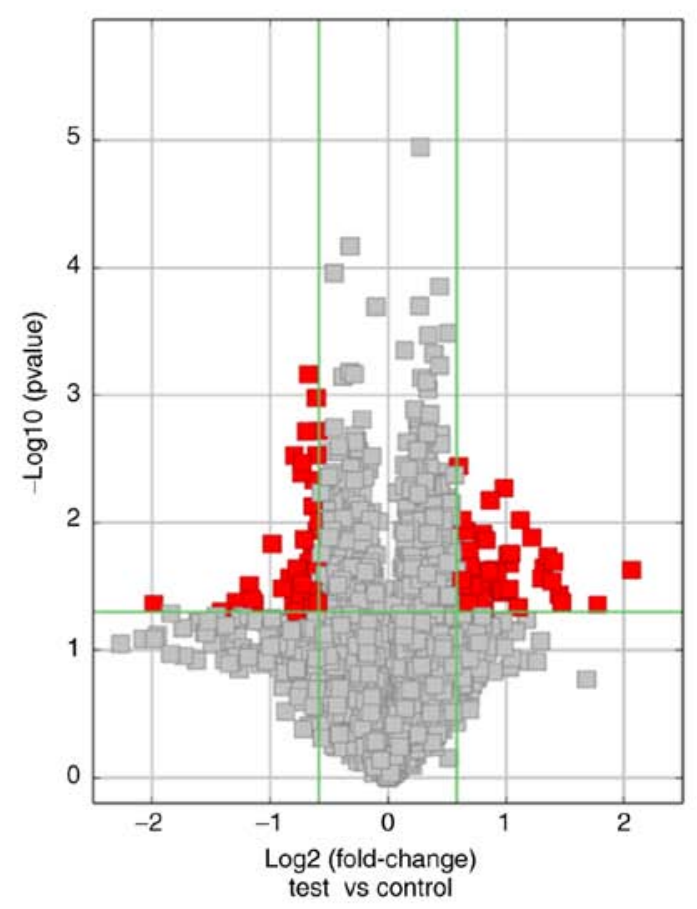

Figure 1. Differential expression of circRNAs between the plasma of patients with IH and healthy children, evaluated using microarray. (A) Box plot indicating the normalized expression values in all six samples. (B) Scatter plot assessing the variation in circRNA expression between the plasma of patients with IH and healthy children. circRNAs above the top green line and below the bottom green line indicate a $>1.5$-fold change in circRNAs between the two groups. (C) Volcano plots visualizing the differential expression of circRNAs. The red dots in the plot represent the significantly differentially expressed circRNAs. (D) Hierarchical clustering analysis of all the differentially expressed circRNAs. Each column in the graph represents a sample, each row represents a circRNA and a tree diagram of the hierarchical clustering analysis of the sample is displayed at the top. The results showed that the expression patterns in the test group and the control group were similar, and there were significant differences between the two groups. IH, infantile hemangioma; circRNA, circular RNA.

hsa_circRNA_103546 and hsa_circRNA_103573, targeted at least 25 different miRNAs. In recent years, the role of circRNAs in vascular diseases has been reported $(21,22)$ and certain miRNAs have been confirmed to be involved in the pathogenesis of IH (23-25). In the present study, three pairs of plasma specimens were first analyzed using a microarray. A total of 72 upregulated and 56 downregulated circRNAs $(\mathrm{FC} \geq 1.5, \mathrm{P}<0.05)$ were identified. Previous studies have analyzed circRNA expression in IH tumor tissues using microarray (10) and RNA-seq (26); however, circRNA expression in plasma samples from $\mathrm{IH}$ patients has not been previously examined. Li et al (27), by comparing a large cohort, determined that microarray is more sensitive and efficient than RNA sequencing (RNA-seq). In addition, certain studies have suggested that plasma samples from cancer patients produce even more accurate results compared with those obtained using tissue samples to study circRNAs for diagnostic applications $(28,29)$. In the present study, a preliminary analysis of the 128differentially expressed circRNAs screened was performed and 6 upregulated and 4 downregulated circRNAs 

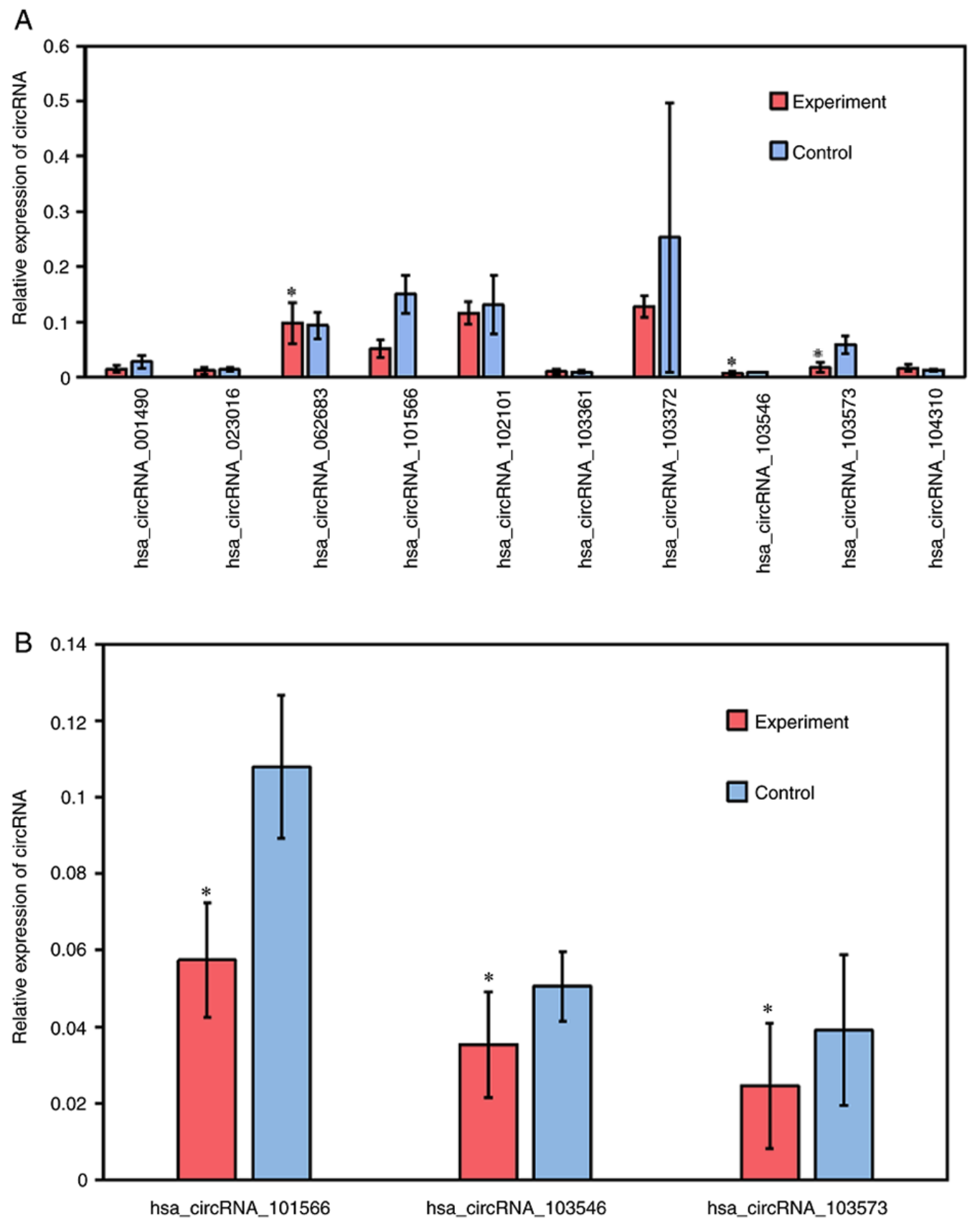

Figure 2. Reverse transcription-quantitative PCR verifying the selected differentially expressed circRNAs. The relative expression of the selected circRNAs in IH was compared to that of control samples. (A) Preliminary validation of 10 selected circRNAs. (B) Extended validation of the three selected circRNAs. If the difference was statistically significant, the result was in accordance with the microarray result. ${ }^{~} \mathrm{P}<0.05$ vs. control. IH, infantile hemangioma; circRNA, circular RNA; hsa, Homo sapiens.

were selected for validation using RT-qPCR, including the 3 downregulated circRNAs hsa_circRNA_101566, hsa_circRNA_103546 and hsa_circRNA_103573. The results were consistent with the microarray results. Studies on circRNAs involved in IH were first performed by our group in 2017 (10), in which microarray analysis detected 234 upregulated and 374 downregulated circRNAs from four pairs of tumor tissue. Subsequently, Li et al (26) identified 124 upregulated and 125 downregulated circRNAs using RNA-seq analysis of three pairs of tumor tissue. However, the circRNAs validated by the two groups are not the same and exhibited less overlap in the experimental results. Of the circRNAs screened for statistically significant differences in the present study, only one (hsa_circRNA_104310) was mentioned in the study by Fu et al (10). The reasons for this were further analyzed. First, as only three or four sample pairs were used for the analysis in the three studies, this led to sample errors of the microarray or RNA-seq results. Furthermore, the circRNAs validated by Li et al (26) were randomly selected and it was not based on any specific strategy to select the most relevant circRNAs in their methods. Generally, it is best to choose the most distinct circRNAs or the circRNAs that may serve an important role in the regulatory network. Finally, while the present study was the first to analyze circRNAs in plasma from pediatric patients with $\mathrm{IH}$, previous studies have analyzed circRNAs in tumor samples. There is likely a difference in circRNA expression 
A

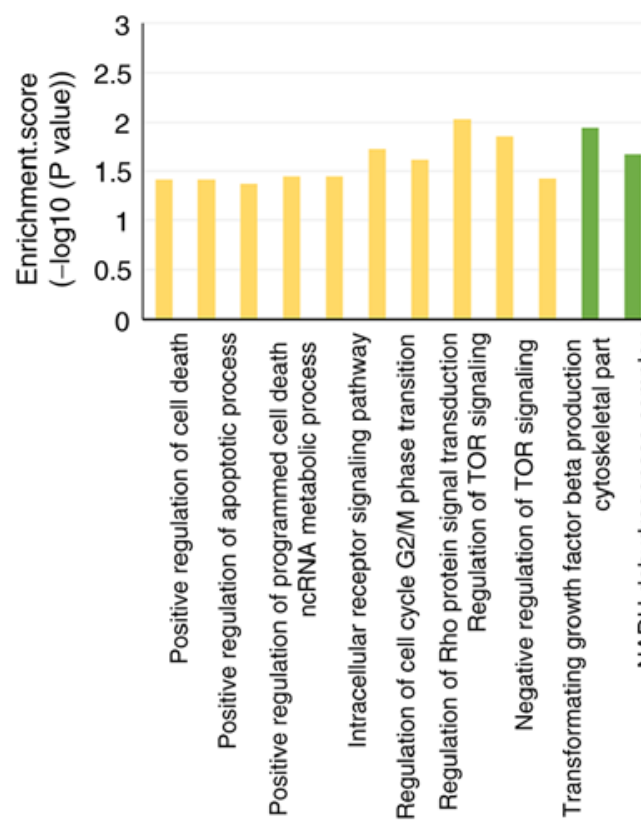

Sig Go terms DE gene

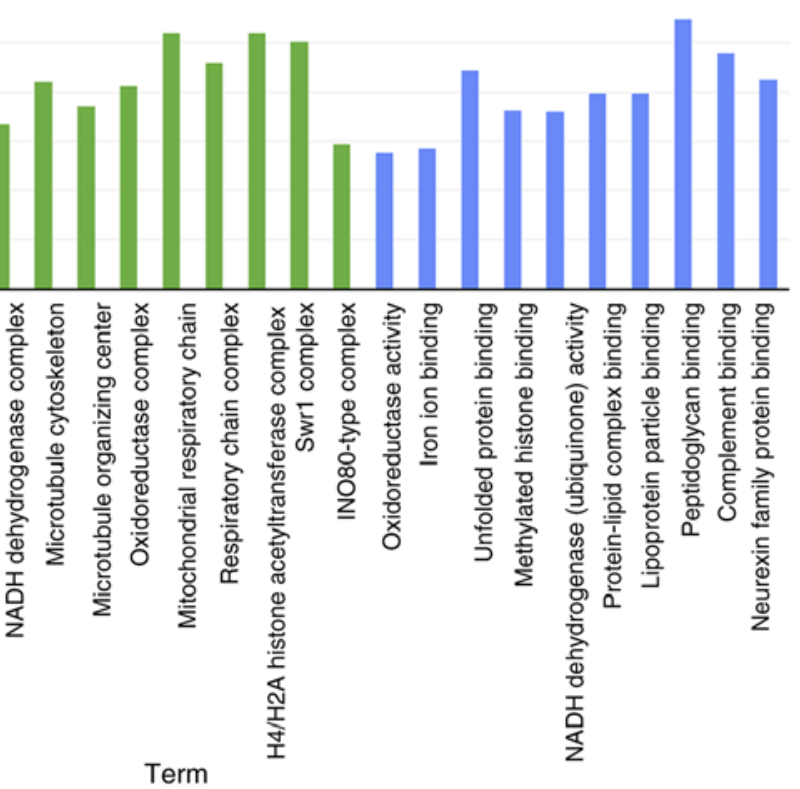

Biological process

Cellular component

Molecular function

B

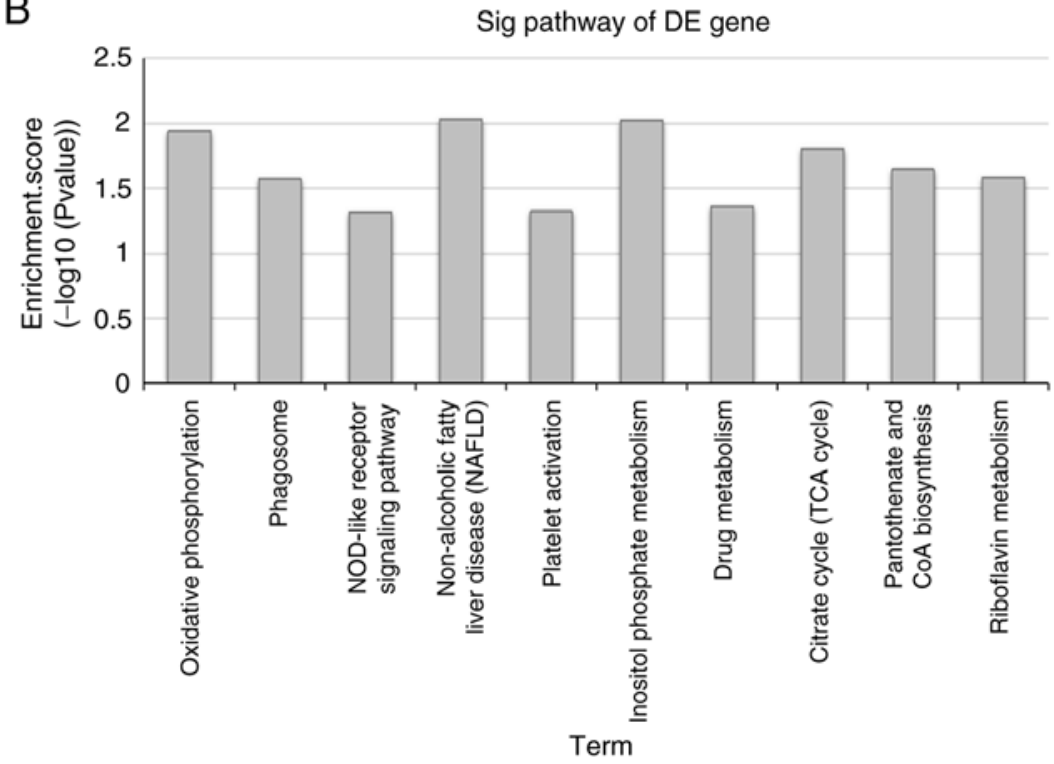

Figure 3. Results of GO and KEGG pathway analyses. (A) Pathways in the three components of the GO analysis. The yellow, green and blue color represents the category molecular function, cellular component and biological process, respectively. (B) KEGG pathway analysis for the downregulated circRNAs. GO, Gene Ontology; KEGG, Kyoto Encyclopedia of Genes and Genomes; DE, differentially expressed; Sig, significant.

between plasma and tumor tissue and this difference should be further explored. These results suggest that the use of different analytical methods and different samples may yield different results and that the development of IH may involve different regulatory pathways in tumor tissue and plasma; these results are consistent with the cell- and tissue-specific expression of circRNAs (30). Therefore, for circRNA investigations in IH, large samples and multicenter studies are required. In addition, the lack of comparison of tumor and plasma samples from the same patient is also a limitation of the present study. In the future, both tumor and plasma specimens from patients will be collected for validation to obtain more convincing results.

A previous study indicated that hsa_miR_520c expression was significantly upregulated in IHs, suggesting that this miRNA is important in IHs (13), whereas bioinformatics analysis (using TargetScan and miRanda) suggested that hsa_circRNA_101566 and hsa_circRNA_103546 are 'miRNA sponges' of hsa_miR_520c. Therefore, targeting of hsa_miR_520c by hsa_circRNA_101566 and hsa_circRNA_103546 may be involved in IH development. Furthermore, certain circRNA-targeted miRNAs (hsa_miR_765, hsa_miR_486, hsa_miR_18a,hsa_miR_10a, hsa_miR_223 and hsa_miR_522) have been indicated to participate in promoting angiogenesis or inducing tumor cell proliferation (14-20) and may be related to angiogenesis and angiogenesis-related disorders in IHs.

GO is a bioinformatics tool originally designed to unify the representation of genes and gene products of various species (31). 

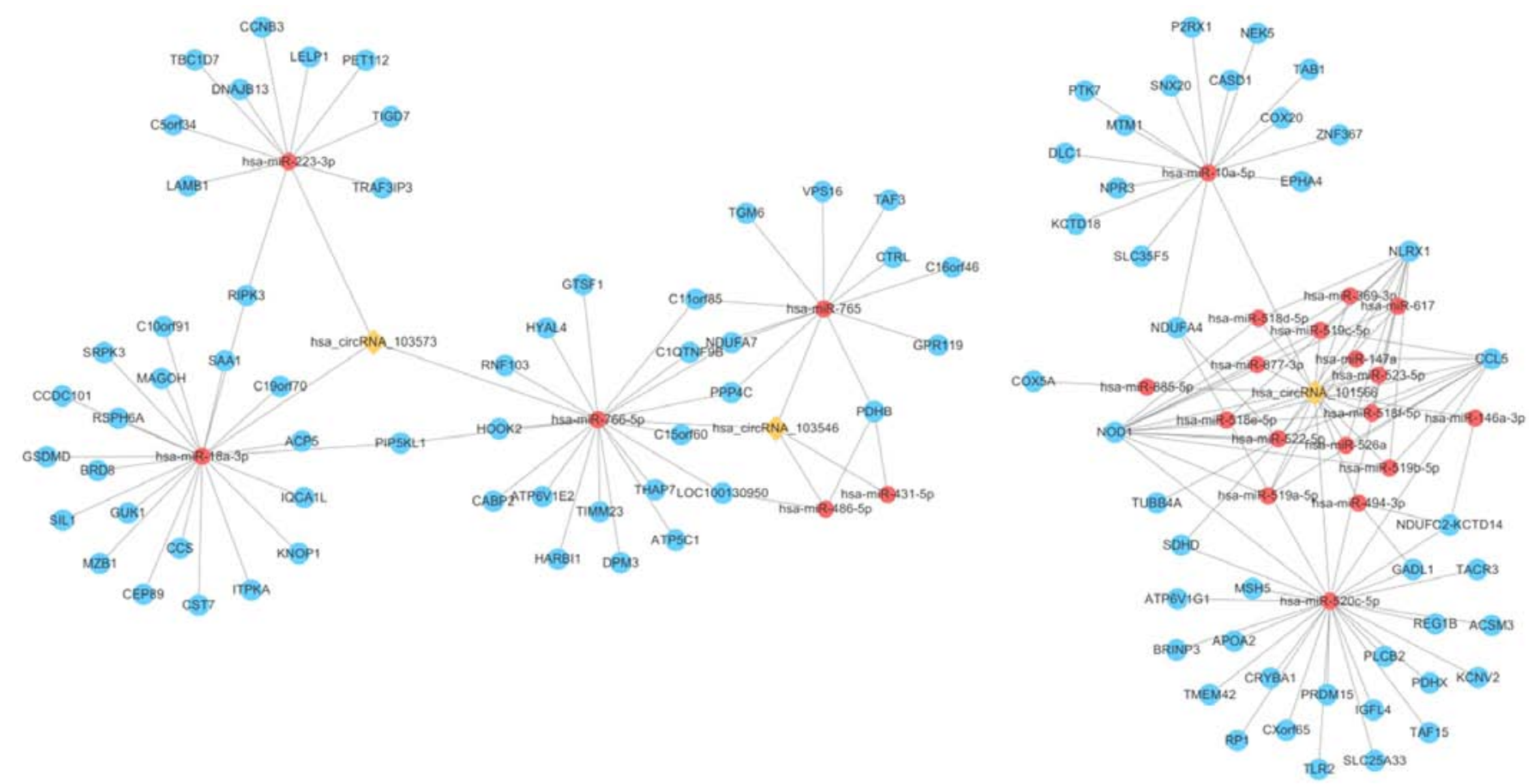

Figure 4. circRNA-miRNA-mRNA network of infantile hemangioma. The circRNA-miRNA-mRNA network was mapped based on the expression profiling results and constructed using Cytoscape software. The yellow, red and blue nodes represent the circRNAs, miRNAs and mRNAs, respectively. circRNA, circular RNA; miRNA, microRNA; hsa, Homo sapiens.

The ontology includes three categories: Cellular component, molecular function and biological process. GO analysis of these target genes, which indirectly regulate the three downregulated circRNAs, revealed their involvement in physiological function that are closely related to the development of IH. Yang et al (32) demonstrated that ras homolog family member A (RhoA), indirectly controlled by hsa_circRNA_101566, is able to regulate cellular apoptosis. In the absence of RhoA, the apoptosis of human umbilical vein endothelial cells induced by TNF- $\alpha$ was inhibited. Conversely, Del Re et al (33) reported that RhoA/Rho kinase activation induces a mitochondrial death pathway and cardiomyocyte apoptosis, which may be correlated with the proliferation of hemangioma-derived endothelial cells during IH proliferation. Of note, this study confirmed that TOR has an important role in the vital activity of cells, while it has been indicated that mammalian target of rapamycin (mTOR) is present in humans. Medici and Olsen (34) demonstrated that rapamycin inhibits the proliferation of hemangioma-derived endothelial cells by decreasing hypoxia-inducible factor-1 (HIF-1) expression in endothelial cells and that the mTOR-related signaling pathway may be one of the mechanisms of IH pathogenesis. Thus, rapamycin is able to inhibit angiogenesis as an mTOR inhibitor and mTOR-related signaling pathways may contribute to the pathogenesis of IH. Certain target genes of hsa_circRNA_101566 are involved in the negative regulation of mTOR signaling, suggesting that mTOR signaling is upregulated and may contribute to proliferation in $\mathrm{IH}$.

KEGG is a knowledge database for systematic analysis of gene function, linking genomic information with higher-order functional information (35). KEGG analysis suggested that pathways, such as the pantothenate and CoA biosynthesis pathways and the NOD-like receptor and citrate cycle pathways, are influenced by the differential expression of the circRNAs. Succinate dehydrogenase (SDH) in the citrate cycle consists of four subunits (36), namely SDHA, SDHB, SDHC and SDHD. The downregulated hsa_circRNA_103546 is able to indirectly regulate the low expression of SDHD. Selak et al (37) reported that the accumulation of succinate due to SDH inhibition led to activation of HIF-1 $\alpha$, which may also be involved in the pathogenesis of IH. In addition, hsa_circRNA_101566 indirectly regulates NOD-like receptor family member X1 (NLRX1), which may be regulated by miR-520c involved in the NOD-like receptor signaling pathway (38), whereas NLRX1 is able to inhibit NF- $\mathrm{kB}$ and thus indirectly inhibit vascular endothelial growth factor (VEGF) (39). It was previously suggested that VEGF contributes to IH development (40) and its downregulation weakens the inhibitory effect on VEGF, which may also be a promising finding in IH. The hypoxia theory, one of the most widely accepted theories regarding the pathogenesis of IHs, states that hypoxic stress triggers the overexpression of angiogenic factors, such as VEGF, via the HIF-1 $\alpha$ pathway $(41,42)$. In support of this, the present study hypothesized that the hsa_circRNA_101566/hsa-miR-520c-5p/NLRX1 signaling axis has a role in the molecular mechanisms of IH development. However, further studies are required to determine the function of circRNAs and circRNA-miRNA-mRNA networks in $\mathrm{IH}$.

In conclusion, the present study was the first, to the best of our knowledge, to detect and profile circRNAs in the plasma of patients with IH. A total of 128 differentially expressed circRNAs, among which 72 were upregulated and 56 downregulated, were screened. Furthermore, three downregulated circRNAs (hsa_circRNA_101566, hsa_circRNA_103546, and hsa_circRNA_103573) were verified using RT-qPCR. 
The roles of differentially expressed circRNAs were explored using GO and KEGG pathway analyses. It was determined that hsa_circRNA_101566, which is able to regulate the mTOR signaling pathway, may be an important regulatory molecule in IH development and that the VEGF signaling pathway may be regulated indirectly by targeting hsa_miR_520c. However, a limitation of the present study was the small number of samples used; further larger studies are required in order to determine whether these circRNAs may be used as biomarkers. Furthermore, functional verification of the roles of the relevant RNA molecules in vitro and in vivo is required. These studies may provide novel ideas for accurate diagnosis and a better understanding of the pathogenesis of $\mathrm{IH}$.

\section{Acknowledgements}

Not applicable.

\section{Funding}

This work was supported by the National Natural Science Foundation of China (grant no. 81873938).

\section{Availability of data and materials}

The datasets generated and/or analyzed during the current study are available from the Gene Expression Omnibus repository (https://www.ncbi.nlm.nih.gov/geo/).

\section{Authors' contributions}

JB was involved with conceptualization and design of the study, study supervision and statistical analysis. ZL, YC performed experimental operations, statistical analysis, data analysis and manuscript writing. ZZ and XL were involved with acquisition of data. RH helped conceptualize design and supervise the study. JB and RH checked and approved the authenticity of the raw data. All authors read and approved the final manuscript.

\section{Ethics approval and consent to participate statement}

This study was approved by the Ethics Committee of Shandong Provincial Hospital (Jinan, China; no. 2018-051) and performed according to the Ethical Guidelines of the Declaration of Helsinki. Written informed consent was obtained from the guardian of each subject.

\section{Patient consent for publication}

Not applicable.

\section{Competing interests}

The authors declare that they have no competing interests.

\section{References}

1. Hoornweg MJ, Smeulders MJC, Ubbink DT and van der Horst CM: The prevalence and risk factors of infantile haemangiomas: A case-control study in the Dutch population. Paediatr Perinat Epidemiol 26: 156-162, 2012.
2. Kilcline $\mathrm{C}$ and Frieden IJ: Infantile hemangiomas: How common are they? A systematic review of the medical literature. Pediatr Dermatol 25: 168-173, 2008.

3. Tollefson MM and Frieden IJ: Early growth of infantile hemangiomas: What parents' photographs tell us. Pediatrics 130: e314-e320, 2012.

4. Chang LC, Haggstrom AN, Drolet BA, Baselga E, Chamlin SL, Garzon MC, Horii KA, Lucky AW, Mancini AJ, Metry DW, et al: Growth characteristics of infantile hemangiomas: Implications for management. Pediatrics 122: 360-367, 2008.

5. Sanger HL, Klotz G, Riesner D, Gross HJ and Kleinschmidt AK: Viroids are single-stranded covalently closed circular RNA molecules existing as highly base-paired rod-like structures. Proc Natl Acad Sci USA 73: 3852e3856, 1976.

6. Hansen TB, Jensen TI, Clausen BH, Bramsen JB, Finsen B, Damgaard CK and Kjems J: Natural RNA circles function as efficient microRNA sponges. Nature 495: 384-388, 2013.

7. Ashwal-Fluss R, Meyer M, Pamudurti NR, Ivanov A, Bartok O, Hanan M, Evantal N, Memczak S, Rajewsky N and Kadener S: circRNA biogenesis competes with pre-mRNA splicing. Mol Cell 56: 55-66, 2014.

8. Du WW, Zhang C, Yang W, Yong T, Awan FM and Yang BB: Identifying and characterizing circRNA-protein interaction. Theranostics 7: 4183-4191, 2017.

9. Li Y, Zheng Q, Bao C, Li S, Guo W, Zhao J, Chen D, Gu J, He X and Huang S: Circular RNA is enriched and stable in exosomes: A promising biomarker for cancer diagnosis. Cell Res 25: 981-984, 2015.

10. Fu C, Lv R, Xu G, Zhang L, Bi J, Lin L, Liu X and Huo R: Circular RNA profile of infantile hemangioma by microarray analysis. PLoS One 12: e0187581, 2017.

11. Johnson CM and Navarro OM: Clinical and sonographic features of pediatric soft-tissue vascular anomalies part 1: Classification, sonographic approach and vascular tumors. Pediatr Radiol 47: 1184-1195, 2017.

12. Livak KJ and Schmittgen TD: Analysis of relative gene expression data using real-time quantitative PCR and the 2(-Delta Delta C(T)) method. Methods 25:402-408, 2001.

13. Strub GM, Kirsh AL, Whipple ME, Kuo WP, Keller RB, Kapur RP, Majesky MW and Perkins JA: Endothelial and circulating C19MC microRNAs are biomarkers of infantile hemangioma. JCI Insight 1: e88856, 2016.

14. Xie BH, He X, Hua RX, Zhang B, Tan GS, Xiong SQ, Liu LS, Chen W, Yang JY, Wang XN and Li HP: Mir-765 promotes cell proliferation by downregulating INPP4B expression in human hepatocellular carcinoma. Cancer Biomark 16: 405-413, 2016.

15. Shi XF, Wang H, Xiao FJ, Yin Y, Xu QQ, Ge RL and Wang LS: MiRNA-486 regulates angiogenic activity and survival of mesenchymal stem cells under hypoxia through modulating Akt signal. Biochem Biophys Res Commun 70: 670-677, 2016.

16. Yang Q, Wang X, Cui J, Wang P, Xiong M, Jia C, Liu L, Ning B, Li L, Wang W, et al: Bidirectional regulation of angiogenesis and miR-18a expression by PNS in the mouse model of tumor complicated by myocardial ischemia. BMC Complement Altern Med 14: 183, 2014.

17. Fei D, Zhang X, Liu J, Tan L, Xing J, Zhao D and Zhang Y: Long noncoding RNA FER1L4 suppresses tumorigenesis by regulating the expression of PTEN targeting miR-18a-5p in osteosarcoma. Cell Physiol Biochem 51: 1364-1375, 2018.

18. Wang X, Ling CC, Li L, Qin Y, Qi J, Liu X, You B, Shi Y, Zhang J, Jiang Q, et al: MicroRNA-10a/10b represses a novel target gene mib1 to regulate angiogenesis. Cardiovasc Res 110: 140-150, 2016.

19. Henn D, Abu-Halima M, Wermke D, Falkner F, Thomas B, Köpple C, Ludwig N, Schulte M, Brockmann MA, Kim YJ, et al: MicroRNA-regulated pathways of flow-stimulated angiogenesis and vascular remodeling in vivo. J Transl Med 17: 22, 2019.

20. Hao Z, Chao Y, Meiyuan C, Zhu L, Se T, Jianxin J and Sun C: MiR-522 contributes to cell proliferation of hepatocellular carcinoma by targeting DKK1 and SFRP2. Tumor Biol 37: 11321-11329, 2016.

21. Li CY, Ma L and Yu B: Circular RNA hsa_circ_0003575 regulates oxLDL induced vascular endothelial cells proliferation and angiogenesis. Biomed Pharmacother 95: 1514-1519, 2017.

22. Boeckel JN, Jaé N, Heumüller AW, Chen W, Boon RA, Stellos K, Zeiher AM, John D, Uchida S and Dimmeler S: Identification and characterization of hypoxia-regulated endothelial circular RNA. Circ Res 117: 884-890, 2015.

23. Bertoni N, Pereira LM, Severino FE, Moura R, Yoshida WB and Reis PP: Integrative meta-analysis identifies microRNA-regulated networks in infantile hemangioma. BMC Med Genet 17: 4, 2016. 
24. Li D, Li P, Guo Z, Wang H and Pan W: Downregulation of miR-382 by propranolol inhibits the progression of infantile hemangioma via the PTEN-mediated AKT/mTOR pathway. Int J Mol Med 39: 757-763, 2017.

25. Li J, Li Q, Chen L, Gao Y, Zhou B and Li J: Competitive endogenous RNA networks: Integrated analysis of non-coding RNA and mRNA expression profiles in infantile hemangioma. Oncotarget 9: 11948-11963, 2018.

26. Li J, Li Q, Chen L, Gao Y and Li J: Expression profile of circular RNAs in infantile hemangioma detected by RNA-Seq. Medicine (Baltimore) 97: e10882, 2018.

27. Li S, Teng S, Xu J, Su G, Zhang Y, Zhao J, Zhang S, Wang H, Qin W, Lu ZJ, et al: Microarray is an efficient tool for circRNA profiling. Brief Bioinform 20: 1420-1433, 2019.

28. Tan H, Gan L, Fan XM, Liu L and Liu S: Diagnostic value of circular RNAs as effective biomarkers for cancer: A systematic review and meta-analysis. Oncotargets Ther 12: 2623-2633, 2019.

29. Li J, Li H, Lv XT, Yang Z, Gao M, Bi Y, Zhang Z, Wang S, Cui Z, Zhou B and Yin Z: Diagnostic performance of circular RNAs in human cancers: A systematic review and meta-analysis. Mol Genet Genom Med 7: e00749, 2019.

30. Chen B and Huang S: Circular RNA: An emerging non-coding RNA as a regulator and biomarker in cancer. Cancer Lett 418 : 41-50, 2018.

31. Yuan F, Pan X, Chen L, Zhang YH, Huang T and Cai YD: Analysis of protein-protein functional associations by using gene ontology and KEGG pathway. Biomed Res Int 2019: 4963289, 2019.

32. Yang L, Tang L, Dai F, Meng G, Yin R, Xu X and Yao W: Raf-1/CK2 and RhoA/ROCK signaling promote TNF- $\alpha$-mediated endothelial apoptosis via regulating vimentin cytoskeleton. Toxicology 389: 74-84, 2017.

33. Del Re DP, Miyamoto S and Brown JH: RhoA/Rho kinase up-regulate Bax to activate a mitochondrial death pathway and induce cardiomyocyte apoptosis. J Biol Chem 282: 8069-8078, 2007.
34. Medici D and Olsen BR: Rapamycin inhibits proliferation of hemangioma endothelial cells by reducing HIF-1-dependent expression of VEGF. PLoS One 7: e42913, 2012.

35. Kanehisa M and Goto S: KEGG: Kyoto encyclopedia of genes and genomes. Nucleic Acids Res 28: 27-30, 2000.

36. Moosavi B, Zhu XL, Yang WC and Yang GF: Molecular pathogenesis of tumorigenesis caused by succinate dehydrogenase defect. Eur J Cell Biol 99: 151057, 2020.

37. Selak MA, Armour SM, MacKenzie ED, Boulahbel H, Watson DG, Mansfield KD, Pan Y, Simon MC, Thompson CB and Gottlieb E: Succinate links TCA cycle dysfunction to oncogenesis by inhibiting HIF-alpha prolyl hydroxylase. Cancer Cell 7: 77-85, 2005.

38. Liu Y, Tang N, Cao K, Wang S, Tang S, Su H and Zhou J: Negative-pressure wound therapy promotes wound healing by enhancing angiogenesis through suppression of NLRX1 via miR-195 upregulation. Int J Low Extrem Wounds 17: 144-150, 2018.

39. Xu W, Li S, Yu F, Zhang Y, Yang X, An W, Wang W and Sun C: Role of Thrombospondin-1 and Nuclear Factor- $\kappa \mathrm{B}$ signaling pathways in antiangiogenesis of infantile hemangioma. Plast Reconstr Surg 142: 310e-321e, 2018.

40. Przewratil P, Sitkiewicz A and Andrzejewska E: Local serum levels of vascular endothelial growth factor in infantile hemangioma: Intriguing mechanism of endothelial growth. Cytokine 49: 141-147, 2010.

41. Drolet BA and Frieden IJ: Characteristics of infantile hemangiomas as clues to pathogenesis: Does hypoxia connect the dots? Arch Dermatol 146: 1295-1299, 2010.

42. Leaute-Labreze C, Prey S and Ezzedine K: Infantile haemangioma: Part I. Pathophysiology, epidemiology, clinical features, life cycle and associated structural abnormalities. J Eur Acad Dermatol Venereol 25: 1245-1253, 2011.

(i) (9) This work is licensed under a Creative Commons Attribution-NonCommercial-NoDerivatives 4.0 International (CC BY-NC-ND 4.0) License. 